\title{
DETECTION OF DISLOCATION LINE BY BAROMETRIC GRADIENT.
}

By S. T. Nakamura.

On the barometric gradient as a secondary cause of the earthquake, Prof. Terada(1) and Mr. Hasegawa ${ }^{(2)}$ already discussed thoroughly. ${ }^{(3)}$ The latter proved that at Gihu the direction of the gradient is always perpendicular to the dislocation line.

If this close relation of them exists at anywhere, by the gradient at the epoch, the direction of dislocation line and also its character in any region may be found approximately, from the direction of the barometric gradient whenever an earthquake occurs. This method is more acculate, if it holds anywhere, than to determine the seismic zone merely statistically by locating a large number of epicentres, and sufficient to secure the existence of seismic zone. For the seismic zone determined by the locating cpicentres is quite uncertain and arbitrary, but our method is more accurate; and in favourable case may determine the approximate direction of the Zone by a few of earthquakes.

I examined 50 earthquakes at Tukuba during the year I904. The results are given in Table I, II and Figure.

The pressures at the epoch when the earthquake occurs are determined graphically from 4 observations at each stations using the six-daily observations at Mito and Utunomiya and hourly obser-

(1) Terada. Proc. Tôkyô Mathematics-Physical Soc. Ser. II Vol. IV. (Igog), P. 454 . (2) Hasegawa. ,
P. I $8 \mathrm{r}$.

(3) Prof. Nagaoka Treated This Problem Mathematically in Proc. Tôkyô Mathematics-Physical Soc. Ser. IT. Vol. VI. (IgL2), P. 208 . 
vations at the Base station at Tukuba. The elevations of these stations are 3 I.I meter, 125.0 meter and 30.0 meter respectively. And Mito is apart from Tukuba about $38 \mathrm{~km}$. and Utunomiya about $44 \mathrm{~km}$.

The brometric gradients at Tukuba are found by Prof, Okada's method as Mr. J Jasegawa did, and taken positively when the pressure at Tukuba is higher than at Mito or Utunomiya. The component gradients T.-M. and T.-U. are given in Table I.

From the result we can easily find that

1) The direction of the gradient at the earthquake epoch is perpendicular to the seismic zone which suggested by Prof. Omori (Pub. of Eqk. Inv. Com. No. II). Prof. Omori suggested this Zone from some theoretical consideration and proved statistically by the distribution of the epicentres. Existence of this Zone may be secured by our agreement, with quite different kind of method. And this is at other hand agrees with the results of Mr. Hasegawa.

2) The second fact is a great fault of my paper: It is the fact that the direction of the gradient coincides to that of annual and monthly mean pressures (Table I and II). But we must remark that the earthquake occurs more frequent in this region, than at the surrounding regions. For example at Mito the number of earthquakes during this year is $I_{4}$ and at Tokyô 8 while it amounts to 50 at this region. Hence we may consider that the above coincidence of the directions will be the cause of frequent occurrence of small earthquakes at this region. I have this opportunity to express my heartful thanks to Prof. Terada, Prof. Okada, Mr. Hasegawa, and Mr. Fudiwhara for their kind guidance and suggestions. 


\section{Table I.}

\begin{tabular}{|c|c|c|c|c|c|c|c|c|c|c|c|}
\hline \multirow{3}{*}{$\begin{array}{c}\text { No. } \\
\text { I }\end{array}$} & \multirow{2}{*}{\multicolumn{2}{|c|}{ Date }} & & & & \multicolumn{3}{|c|}{ Barometric Pressure } & \multicolumn{2}{|c|}{ Barometric Gradient } & \multirow[b]{2}{*}{$\begin{array}{l}\text { Direction o } \\
\text { Greatlitent }\end{array}$} \\
\hline & & & & Time & & Mito & $\begin{array}{l}\text { Utuno- } \\
\text { nilya }\end{array}$ & Tukuba & $\mathrm{T}-\mathrm{M}$ & $\mathrm{r},-\mathrm{U}$ & \\
\hline & I & 3 & $t^{h}$ & $58 \mathrm{~m}$ & $\mathrm{p}$ & $75 x .9$ & $7 \pm 0.9$ & 752.5 & +0.016 & +0.036 & $\mathrm{~N} 4^{2} \mathrm{~W}$ \\
\hline 2 & & 8 & 3 & 25 & $\mathbf{a}$ & 65.3 & 65.0 & 65.5 & +0.003 & +0.011 & $\mathrm{NI}_{5} \mathrm{VH}$ \\
\hline 3 & & I6 & 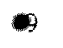 & 2 & $\mathrm{p}$ & $6 \mathrm{r} .2$ & 60.4 & $6 \mathrm{r} .5$ & +0.008 & +0.025 & $N \mathrm{II}^{2} \mathrm{~W}$ \\
\hline 4 & & 16 & IO & 39 & 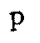 & $6 \mathrm{r} .2$ & 60.5 & $6 \mathrm{r} .5$ & +0.005 & +0.023 & $N g^{*} \mathrm{~W}$ \\
\hline 5 & & 17 & II & 29 & & $59 \cdot 4$ & 58.8 & 50.5 & +0.003 & +0.016 & $N$ Is' $\mathrm{W}$ \\
\hline 6 & & 24 & $\xi$ & 22 & & $66 \cdot 3$ & 65.2 & 65.6 & -0.013 & -0.018 & $\mathrm{~S} I 7^{\circ} \mathrm{WV}$ \\
\hline 7 & & 29 & 2 & 32 & 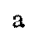 & 69.9 & 70.4 & 69.7 & -0.005 & -0.016 & $S 10^{\circ} \mathrm{E}$ \\
\hline 8 & & $3 \mathrm{I}$ & Io & so & 6 & 61.6 & 61.8 & 6 I.7 & +0.003 & -0.002 & $S 76^{3} \mathrm{E}$ \\
\hline 9 & II & 7 & 3 & 17 & a & 69.1 & 69.6 & 69.7 & +0.016 & +0.002 & $N 51^{\circ} \mathrm{E}$ \\
\hline 10 & & 9 & 7 & 55 & $p$ & 62.1 & 62.2 & 62.3 & -0.005 & +0.002 & $\mathrm{~N} 87^{\circ} \mathrm{W}$ \\
\hline I I & & IO & 0 & rg & 3 & 63.6 & 63.9 & 63.9 & -0.008 & 0.000 & $=62^{\circ} \mathrm{W}$ \\
\hline I 2 & & II & 9 & 32 & 5 & 59.7 & 60.2 & 61.0 & +0.034 & +0.013 & $N 3+E$ \\
\hline I 3 & & I 2 & 3 & I 9 & & 60.9 & 60.4 & 60.6 & -0.008 & +0.005 & $N 37 \mathrm{~W}$ \\
\hline 14 & & I6 & S & $\mathrm{I}_{3}$ & $p$ & $63 \cdot 3$ & 63.6 & 63.5 & +0.005 & -0.002 & $\triangle 65 \mathrm{E}$ \\
\hline$I_{5}$ & & I7 & 3 & 50 & $\xi$ & $\vdots 7.5$ & 5.68 & 56.9 & $-0.0 \mathrm{r} 6$ & +0.002 & $\mathrm{~S} ; 0 \mathrm{~W}$ \\
\hline 16 & III & 3 & 9 & 13 & $\mathrm{r}$ & 66.5 & 6.70 & $66 .+1$ & -0.003 & $-0.0 r_{t}$ & $S \mathrm{I} \mathrm{B}^{2} \mathrm{E}$ \\
\hline I 7 & & 4 & 3 & 23 & $\mathrm{p}$ & 69.0 & 59.8 & 69.0 & 0.000 & -0.18 & $S 2 S^{\circ} \mathrm{E}$ \\
\hline Is & & $\varepsilon$ & 3 & +2 & 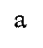 & 71.7 & 71.9 & 70.9 & $-0.02 \mathrm{~T}$ & -0.023 & $\mathrm{~S} I+\mathrm{W}$ \\
\hline 19 & & $\mathrm{r} 2$ & 8 & 49 & $\mathrm{p}$ & $65 \cdot 4$ & 65.4 & 64.9 & -0.13 & $-0.01 \mathrm{I}$ & $521^{\circ} 11$ \\
\hline 20 & & It & 9 & 43 & 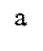 & 66.7 & 67.2 & 66.2 & -0.013 & -0.023 & 5211 \\
\hline $2 I$ & & 25 & 3 & 35 & $\mathrm{p}$ & 53.7 & $\$ 2.5$ & 32.4 & -0.034 & -0.92 & $S=0 \quad W$ \\
\hline 22 & & 26 & 3 & 30 & 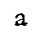 & $56 . I$ & 56.4 & 56.3 & +0.005 & $-0,002$ & $N S: E$ \\
\hline 23 & & 30 & I & 46 & $\mathrm{p}$ & $6+7$ & 64.4 & 64.5 & -0.905 & $\div 0.002$ & $S 85^{\circ} \mathrm{W}$ \\
\hline 24 & IV & 19 & $s$ & 20 & & 58.7 & 58.4 & 59.1 & $+0.0 \mathrm{II}$ & $\div 0.016$ & $N 5 \mathrm{E}$ \\
\hline 25 & & 20 & 9 & 47 & $\mathrm{P}$ & $6 \mathrm{r} .5$ & $6 \mathrm{r} \cdot 3$ & 61.5 & 0.000 & +0.005 & $\mathrm{~N} 2 \mathrm{~W} \mathrm{~W}$ \\
\hline 26 & & 24 & 8 & $2+$ & $P$ & 65.2 & 65.0 & 65.2 & 0.000 & +0.005 & N.5 11 \\
\hline 27 & & 27 & 3 & I2 & a & 59.1 & $59 \cdot 5$ & 58.6 & -0.013 & -0.020 & $S 511$ \\
\hline 28 & V & $I_{4}$ & $=$ & 58 & $\mathrm{P}$ & 62.6 & 62.6 & 62.4 & -0.005 & -0.005 & $\$ 2011$ \\
\hline 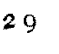 & & 27 & 8 & 17 & P & 64.1 & $64 \cdot 3$ & 64.2 & +0.003 & -0.002 & $\mathrm{~S} 76^{\circ} \mathrm{E}$ \\
\hline 30 & & 30 & 4 & 13 & $\mathrm{p}$ & 57.5 & 57.0 & 56.6 & -0.024 & -0.009 & $S 4 I^{2}$ \\
\hline $3 I$ & II & 7 & 6 & I.4 & a & 52.4 & 52.1 & 52.4 & 0.000 & \pm 0.007 & $N 28^{\prime}$ \\
\hline 32 & VII & 5 & ro & 54 & a & 59.0 & 59.2 & 58.2 & $-0.02 \mathrm{r}$ & -0.023 & $S I f=W$ \\
\hline
\end{tabular}




\begin{tabular}{|c|c|c|c|c|c|c|c|c|c|c|c|c|}
\hline \multirow{3}{*}{$\begin{array}{l}\text { No. } \\
33\end{array}$} & \multirow{2}{*}{\multicolumn{2}{|c|}{ Date }} & \multirow{2}{*}{\multicolumn{3}{|c|}{ Time }} & \multicolumn{3}{|c|}{ Rarametric Pressure } & \multicolumn{2}{|c|}{ Barometric Gradient } & \multirow{2}{*}{\multicolumn{2}{|c|}{$\begin{array}{l}\text { Direction of } \\
\text { Gradient }\end{array}$}} \\
\hline & & & & & & Mito & $\begin{array}{l}\text { Utuno- } \\
\text { miya }\end{array}$ & Tukuba & T. $-\mathbf{M}$ & T. - U. & & \\
\hline & & 3 & $7^{h}$ & $2\left[^{12}\right.$ & $\mathrm{a}$ & $759 \cdot 5$ & $759 \cdot 3$ & 759.0 & -0.013 & $-0,007$ & S $35^{\prime}$ & W \\
\hline 34 & VIII & 20 & Io & $5 I$ & $\mathrm{p}$ & 6I.9 & 62.2 & $6 \mathrm{I} .9$ & 0.000 & -0.007 & S $28^{\circ}$ & $E$ \\
\hline 35 & IX & 23 & 8 & 12 & $\mathrm{p}$ & 59.4 & 59.6 & 59.4 & 0.000 & -0.005 & S $28^{\circ}$ & $\mathbf{E}$ \\
\hline 36 & $\mathrm{x}$ & 4 & 8 & 42 & a & 58.1 & 58.3 & 58.7 & +0.016 & +0.009 & $\mathrm{~N} 32^{\circ}$ & $\mathbf{E}$ \\
\hline 37 & & 4 & 9 & I 8 & 4 & 58.1 & $5^{8} .2$ & $5^{8.5}$ & +0.011 & +0.007 & $\mathbf{N} 30^{\circ}$ & $\mathbf{E}$ \\
\hline 38 & & 6 & 9 & 40 & a & 52.7 & 52.6 & 52.4 & -0.058 & -0.005 & $\mathrm{~S} 34^{\circ}$ & W \\
\hline 39 & & 6 & 10 & 21 & a & 53.2 & 52.9 & 52.5 & $-0,018$ & -0.009 & S $36^{\circ}$ & W \\
\hline 40 & & 6 & IO & 39 & a & 53.4 & 53.0 & 52.5 & -0.024 & $-0.01 \mathrm{I}$ & S $37^{\circ}$ & W \\
\hline 4 & & 6 & II & 57 & a & 53.9 & 53.5 & 52.5 & -0.037 & -0.023 & $\operatorname{S~} 30^{\circ}$ & w \\
\hline 42 & & I6 & 8 & 26 & $\mathrm{p}$ & 63.5 & 64.2 & 62.7 & $-0.02 x$ & -0.034 & $\mathrm{~S} \quad 3^{3}$ & w \\
\hline 43 & XI & 8 & II & 47 & a & 58.6 & 57.9 & $59 \cdot 3$ & +0.018 & +0.032 & $\mathrm{~N} \quad 2^{\circ}$ & E \\
\hline 44 & & Io & 6 & 49 & $\mathrm{p}$ & 65.9 & $66 . I$ & 65.5 & $-0.01 \mathrm{I}$ & -0.014 & $\mathrm{~S} 10^{\circ}$ & w \\
\hline 45 & & 18 & 6 & 16 & $\mathrm{p}$ & $71 \cdot 7$ & 71.8 & 71.8 & +0.003 & 0.000 & $\mathrm{~N} 62^{\circ}$ & $\mathrm{E}$ \\
\hline 46 & & 20 & 4 & 14 & $\mathrm{P}$ & 66.0 & $66 . x$ & 66.0 & 0.000 & -0.002 & S $28^{\circ}$ & $\mathbf{E}$ \\
\hline 47 & & 22 & 6 & 56 & a & 60.1 & 60.6 & $6 u, 6$ & +0.013 & 0.000 & $\mathrm{~N} 62^{\circ}$ & $\mathrm{E}$ \\
\hline $4^{8}$ & & 22 & Io & 40 & $\mathrm{p}$ & $54 \cdot 5$ & 55.2 & 54.3 & -0.005 & -0.020 & $\mathrm{SI} 4^{\circ}$ & $E$ \\
\hline 49 & & 27 & 3 & 4 & a & $6 \mathrm{r} \cdot 3$ & $6 r .9$ & 62.1 & +0.021 & +0.005 & $\mathrm{~N} 50^{\circ}$ & $\mathrm{E}$ \\
\hline 50 & & 27 & II & 7 & $\mathrm{p}$ & 66.3 & 66.5 & 66.2 & -0.003 & -0.007 & $7^{\circ}$ & $\mathrm{E}$ \\
\hline & & & & & & 60.9 & GI.I & 60.7 & -0.005 & -0.009 & $I^{\circ}$ & w \\
\hline
\end{tabular}

\section{Table II.}

\begin{tabular}{|c|c|c|c|c|}
\hline January $\ldots \ldots \ldots \ldots$ & $63 . \mathrm{x}$ & 62.9 & 0.000 & -0.005 \\
\hline ebruary ........ & $6+3$ & 64.2 & 0.000 & -0.00 \\
\hline March ................6.6.8 & $63 \cdot 1$ & 62.6 & -0.005 & \\
\hline$\ldots \ldots .63 \cdot 7$ & $63 \cdot 7$ & 63.5 & -0.005 & $-0 . \infty$ \\
\hline 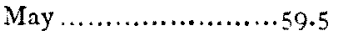 & 69.6 & $59 \cdot 3$ & -0.005 & -0.00 \\
\hline$\ldots \ldots 57.6$ & 57.6 & $57 \cdot 3$ & -0.008 & -0.00 \\
\hline$\ldots \ldots \ldots, 57.5$ & 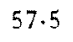 & $57 \cdot 0$ & -0.013 & $-0.01 \mathrm{I}$ \\
\hline$\ldots \ldots \ldots 5^{3} \cdot 5$ & 8.6 & 58.3 & -0.005 & -0.007 \\
\hline September & 50.0 & 57.9 & -0.018 & -0.016 \\
\hline$\ldots \ldots \ldots \ldots \ldots 62.0$ & $62 \cdot 3$ & $6 \mathbf{r} .6$ & $-0.0 \mathrm{Ix}$ & -0.016 \\
\hline November .............91.4 & $6 \mathrm{r} \cdot 7$ & 01.4 & 0.000 & -0.007 \\
\hline December & 62.7 & 62.6 & +0.008 & $-0,0$ \\
\hline
\end{tabular}

